\title{
EVALUACIÓN DE LA COMERCIALIZACIÓN DEL TANGELO (Citrus reticulata $x$ Citrus paradasi) CON MANEJO POST COSECHA EN EL ANEXO DE SANTA ROSA DE CASHINGARI, SATIPO
}

\author{
EVALUATION OF TANGELO COMMERCIALIZATION (Citrus reticulata $x$ Citrus paradasi) WITH \\ POSTHARVEST MANIPULATION IN SANTA ROSA DE CASHINGARI COMMUNITY SATIPO
}

\author{
Angélica Castro Garay', Edgar Rafael Acosta López²
}

\begin{abstract}
RESUMEN
Los factores que intervienen en la post cosecha del Tangelo son la organización, extensión agrícola, accesibilidad al crédito agrario y la comercialización. El costo de producción promedio del Tangelo por hectárea sin post cosecha es de S/ 4 064,00 y la utilidad por campaña oscila entre S/ 818,00 a S/1 853,00 con una rentabilidad desde $20,54 \%$ hasta $44,68 \%$. El costo de post cosecha promedio del Tangelo por hectárea es de $\mathrm{S} / 18$ 912,00 con una utilidad desde S/ 4999,00 hasta S/ 7459,00 con una rentabilidad de 28,93\% hasta $36,38 \%$ en la primera campaña. Para la segunda campaña la rentabilidad del tangelo con poscosecha se incrementó $61,49 \%$. Durante el tratamiento post cosecha se obtiene: $5 \%$ de descarte, $30 \%$ de calidad primera, $45 \%$ de calidad segunda y $20 \%$ calidad tercera. La presentación del producto es en cajas de cartón corrugado de 40,50 y 60 unidades según la calidad. Con la contrastación estadística se puede afirmar con $95 \%$ de seguridad que la rentabilidad es mayor en el Tangelo con post cosecha a partir de la segunda campaña. Se concluye que con la aplicación del tratamiento post cosecha en el Tangelo se logra incrementar la rentabilidad económica del agricultor el cual tendrá impacto en la ampliación de su frontera agrícola, alimentación, educación, vestimenta y salud.
\end{abstract}

Palabras clave: Tangelo, post cosecha, cadena productiva.

\begin{abstract}
The post harvest factors of Tangelo are: the organization, agricultural extension, accessibility to agricultural credit and commercialization. The production cost average of tangelo per hectare is $\mathrm{S} /$ 4064,00 and the utility by campaign is between S/ 818,00 and $S / 1853,00$ with a yield from $20,54 \%$ to $44,68 \%$. The post harvest cost average of Tangelo per hectare is S/18 912,00 a utility from S/ 4 999,00 to $S / 7459,00$ with a yield from $28,93 \%$ to $36,38 \%$ for the first campaign. In the second campaign, the yield of tangelo with post harvest manipulation increased $61,49 \%$. It was been obtained $30 \%$ first quality, $45 \%$ second quality, $20 \%$ third quality and $5 \%$ of discard (low quality tangelo) during post harvest treatment. The product is presented in corrugated cardboard box of 40, 50 and 60 units according to the quality. According to the statistical contrast through the T student test, it is possible to affirm with $95 \%$ of security that the utility is more in tangelo with post harvest manipulation than in tangelo without post harvest manipulation from the second campaign. It's concluded that the post harvest manipulation increases the economic yield of the agriculturist, which will have impact in the extension of its agricultural border, food, education, dressing and health.
\end{abstract}

Key words: Tangelo, post harvest, productive chain

${ }^{2}$ Facultad de Ingeniería en Industrias Alimentarias. Universidad Nacional del Centro del Perú. 


\section{INTRODUCCIÓN}

El tratamiento post cosecha de un producto agrícola es una estrategia integrada en la cual intervienen muchos factores desde las labores de precosecha hasta que el producto llegue al consumidor, generando una mejora en la productividad y mejor rentabilidad. La articulación de los servicios conexos que participan en el agro, la organización de los productores, la industria y el mercado, forman una cadena productiva que será una alternativa para el productor rural minifundista (1).

En la selva central del país, el productor de cítricos tiene serios problemas con la producción y mercado, baja producción y bajos precios, que ocasiona en los productores conformismo y tiene consecuencias en la alimentación, salud, educación, vestimenta y en la ampliación de su frontera agrícola.

El Tangelo es un cítrico jugoso, de sabor y color agradable que tiene características físicas, químicas y nutricionales aceptables por el consumidor (2). El manejo post cosecha a través de alianzas estratégicas propiciara el desarrollo de la agroindustria rural y través de un trabajo planificado, organizado, dirigido y controlado se incrementara la productividad y la rentabilidad económica.

\section{MATERIAL Y MÉTODOS}

La investigación se realizó en el Anexo de Santa Rosa de Cashingari del distrito y provincia de Satipo. Para evaluar los factores que intervienen en la post cosecha se aplicó encuestas a 33 agricultores de Tangelo, cuyas plantaciones tienen edades desde 02 a 17 años. El ensayo de manejo post cosecha se realizó en una muestra estratificada de 04 agricultores cuyas plantaciones tienen edades entre 8 a 12 años con una extensión de cultivo de 1 hectárea.

La rentabilidad se determinó con la utilidad con respecto al costo de producción (3) y para la comparación de medias de las rentabilidades (sin post cosecha y con post cosecha) se utilizó la prueba de "t" Student a un $\square=0,05$ con 6 grados de libertad y un valor crítico de $-2,447$.

\section{RESULTADOS}

El sistema de comercialización del tangelo se inicia cuando el intermediario visita los campos de cultivo solicitando el producto, en una cantidad determinada y a un precio que él oferta; si le conviene al productor se realiza la transacción. El precio durante la primera campaña en el año 2003 varía desde $\mathrm{S} / .4,00$ hasta 12,00 nuevos soles por cien unidades. El intermediario antes de recoger el producto verifica la calidad y cantidad y si no reúne las condiciones del contrato disminuye el precio y el agricultor tiene que aceptar porque realizó la cosecha y tiene que asumir pagos de personal. El producto se transporta a los mercados de Lima, Huancayo, Chiclayo, Trujillo, Arequipa y Otros.

El Tangelo al Ilegar a Lima antes de ingresar al Mercado de Frutas $\mathrm{N}^{\circ} 2$, interviene otro intermediario o comisionista que es el encargado de clasificar y procesar el producto y coloca una etiqueta que le conviene y que no corresponde al lugar de procedencia para ser expendido al consumidor. Los intermediarios tienen poder $y$ manejan los precios en los mercados especialmente los mayoristas, ya que son los que especulan y determinan una escasez ficticia. Los consumidores del producto son comerciantes mayoristas, minoristas, bodegueros, juguerías y amas de casa. El sistema de comercialización comienza en la chacra (con el productor) y termina con el consumidor, entre estos dos polos se realizan por lo menos tres transacciones, fuera de los transportistas y otros agentes que prestan servicios en la comercialización, donde existen por lo menos dos comerciantes intermedios. En la Figura 1, se presenta la estructura de la comercialización.

Figura 1. Estructura de comercialización del Tangelo

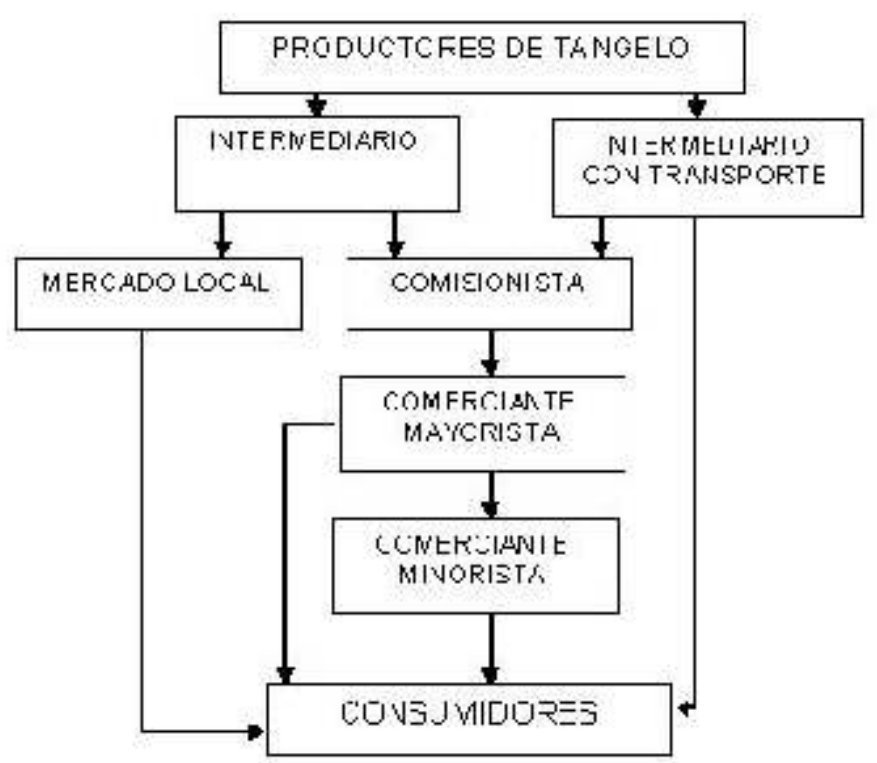

Figura 2, se presenta el árbol de limitaciones en donde se aprecian los factores más importantes detectados como limitantes para el desarrollo de la cadena productiva del Tangelo. 


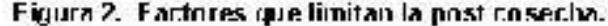

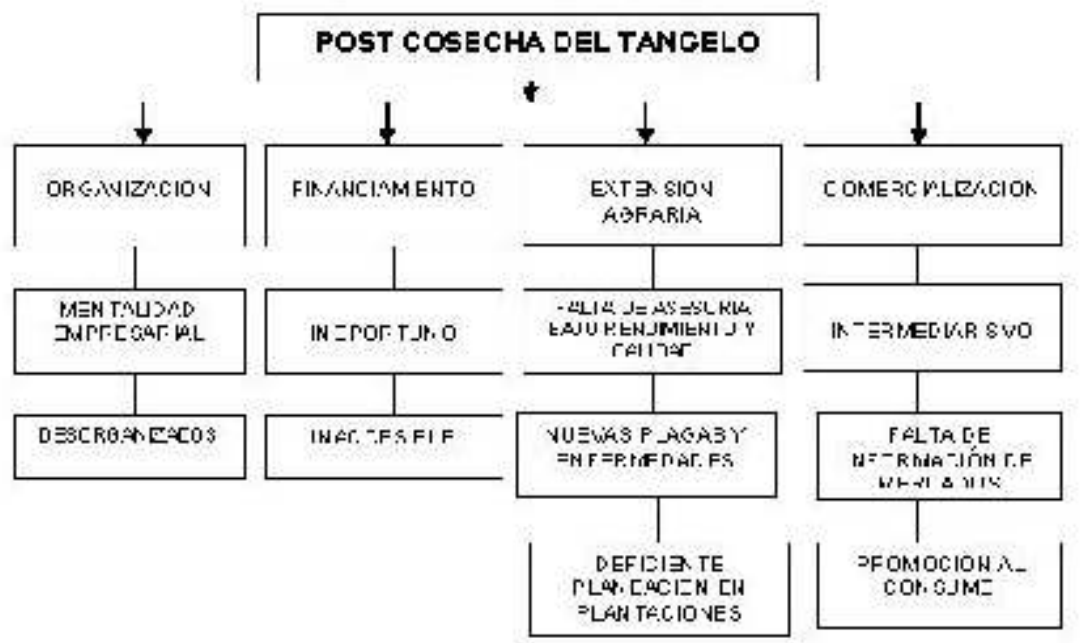

$32,72 \%$ y para el Tangelo con post cosecha (TCPC) de $32,78 \%$ con desviaciones estándar de 10,39 y 3,163 respectivamente. Para la segunda campaña fue TSPC de $32,72 \%$ y para (TCPC) de $61,49 \%$ con desviaciones e stánd a r d e 10,30 y 4,69 respectivamente.

El (TCPC) se presento en cajas de cartón corrugado de 40, 50 y 60 unidades. En el tratamiento post cosecha se encontró que en un lote de 100000 unidades: el $30 \%$ es calidad primera, $45 \%$ segunda, el $20 \%$ calidad tercera y el $5 \%$ es 10,30 y 4,69 respectivamente.
La edad de las plantaciones de Tangelo: 4 agricultores tienen plantas con edades menores a 5 años sin producción, 7 entre 5 a 8 años, 6 de 8 a 12 años, 5 de 12 años y 11 mayor a 15 años.

En la Tabla 1, se presenta el número de plantas de Tangelo/ha y las unidades producidas por planta.

Tabla 3. Utilidad obtenida en el Tangelo con post cosecha

\begin{tabular}{|c|c|c|c|c|c|c|}
\hline \multirow[b]{2}{*}{ Pmanirer } & \multicolumn{3}{|c|}{ Pri mera Zampaña (Sl.) } & \multicolumn{3}{|c|}{ Segunda Camsaña ( $S /$.) } \\
\hline & $\begin{array}{c}\text { Costo da } \\
\text { pmrintrión }\end{array}$ & $\begin{array}{l}\text { hyreso } \\
\text { mrwantas }\end{array}$ & Iltilidar & $\begin{array}{l}\text { Costo do } \\
\text { Pronti rrińn }\end{array}$ & $\begin{array}{c}\text { lingeso } \\
\text { prr went=s }\end{array}$ & I til הar \\
\hline 1 & 20507,50 & 27064,00 & 7450,50 & 16754,50 & 27064,03 & 11200,50 \\
\hline 3 & $1 \exists<86 \leq 0$ & 26104,00 & $E 61750$ & 15986.50 & 26104,03 & 1011750 \\
\hline 4 & $153 / 6, L U$ & 24230,00 & $\leqslant 854,100$ & 15 126 UU & $2<23 L, U J$ & y $104, U$ \\
\hline Promedio & $13912, \mathrm{CO}$ & & E 232,00 & 15537,00 & & $9 \in 07.50$ \\
\hline
\end{tabular}

Tabla 1. Múmera de plaitasilia y unidades producidas le tangeloiplanto.

\begin{tabular}{cccr}
\hline Encuestados & N' Je plantas & Encuestadcs & $\begin{array}{c}\text { I.Inidades } \\
\text { pruduciuts }\end{array}$ \\
15 & 150 & 4 & \\
13 & 180 & 13 & 1 uUL -1200 \\
5 & 200 & 13 & $000-1000$ \\
& & $F$ & 800 \\
\hline 33 & & & \\
\hline
\end{tabular}

El manejo del cultivo de las plantaciones de Tangelo es artesanal y emplea una tecnología baja. En las Tablas 2 y 3 , se presentan las utilidades obtenidas.

Tabla 2. Utilidad obtenida en el Tangelo sin post cosecha

\begin{tabular}{|c|c|c|c|}
\hline Productor & $\begin{array}{c}\text { Costo co } \\
\text { Prodtcción }\end{array}$ & $\begin{array}{c}\text { Ingrezo } \\
\text { jor } \\
\text { ventas }\end{array}$ & Utilidad \\
\hline 1 & 4147,00 & 6000,00 & 1853,00 \\
\hline 2 & $3 y 82, w$ & 4 EUU,UU & $818, \omega$ \\
\hline 3 & 4092,00 & $5 \in 00,00$ & $1508, \infty$ \\
\hline 4 & 4037,00 & 5200,00 & $1163, \infty$ \\
\hline Promedio & 54034,50 & & 1335,50 \\
\hline
\end{tabular}

rentabilidad promedio para la primera campaña para el Tangelo sin post cosecha (TSPC) es de
El (TCPC) se presento en cajas de cartón corrugado de 40, 50 y 60 unidades. En el tratamiento post cosecha se encontró que en un lote de 100000 unidades: el 30\% es calidad primera, $45 \%$ segunda, el $20 \%$ calidad tercera y el $5 \%$ es descarte. El producto debe tener las siguientes características: estado de madurez pinton (coloración de la cáscara 75\% amarillo naranja), de 8 a 8,5 ${ }^{\circ}$ Brix, \% de Acidez 0.60 0.66 y un índice de Madurez de 12.87 a

13.33.

\section{DISCUSIÓN}

Los productores de Tangelo deben enfrentar dos aspectos cruciales que son: capital para financiar la producción y el mercado donde colocar el producto en condiciones que permitan recuperar lo invertido y obtener ganancias. La accesibilidad al crédito por los pequeños productores tiene restricciones como: organización en cadena productiva, superficie cultivada de 3 ha (requisitos mínimos). El mercado es una incertidumbre. $Y$ por ambas razones no se invierte en tecnología y asistencia técnica, los rendimientos son bajos y no se logra organizar y planificar la producción. En la comercialización 
tener un sistema de información que permita una mejor toma de decisión por parte de los agentes que participan en la cadena de comercialización (transparencia de mercado).

Los productores de Tangelo deben organizarse en asociaciones empresariales para ofertar la venta futura e integral de sus cosechas. Con el acopio asociativo de las cosechas de 20 o 30 productores se puede obtener mejores precios y, al mismo tiempo, aprovechar las ventajas de la economía de escala para reducir gastos en la cadena de servicios que deben ser contratados. Entre ellos, la compra en cantidad de insumos, la asesoría técnica, el embalaje y el transporte.

Las edades de las plantaciones de Tangelo en producción se encuentra entre 5 a 15 años, el número de plantas/ha oscila desde 150 a 200. Las plantaciones de Tangelo en la Selva Central requieren de mayor distanciamiento por el desarrollo de las copas, se recomienda $10 \mathrm{~m}$ entre hileras y $8 \mathrm{~m}$ entre plantas y técnicamente se debe tener de 140 a 150 plantas/ha (4), en 18 agricultores la densidad de las plantaciones esta por encima de la recomendación técnica, que significa diferencias en el manejo de plantaciones. La producción en promedio por planta es de 800 unidades que es considerado como baja producción si se tiene en cuenta la edad promedio de las plantas de 10 a 12 años y es en esta edad donde la planta tiene su mayor producción de 1500 a 2000 unidades/planta (5). La baja producción se debe al manejo inadecuado en pre cosecha.

Al comparar los promedios de las rentabilidades del (TSPC) y (TCPC) se puede afirmar con $95 \%$ de seguridad que la rentabilidad del (TCPC) es mayor a la rentabilidad (TSPC) a partir de la segunda campaña. En la primera campaña no existe diferencia entre los promedios de las rentabilidades obtenidas por la mayor inversión en la adquisición de insumos. Se concluye que al comercializar Tangelo con post cosecha se logra incrementar la rentabilidad económica del agricultor el cual tendrá impacto en la ampliación de su frontera agrícola, alimentación, educación, vestimenta y salud

\section{REFERENCIAS BIBLIOGRÁFICAS}

1. Flores A. Manejo post cosecha de frutas y hortalizas en Venezuela. San Carlos: UNELLEZ; 1997

2. Morín Ch. Cultivo de Cítricos. Lima: Universidad Nacional Agraria La Molina Facultad Agronomía; 1965.

3. Espíritu D. Análisis comparativo de costos de producción entre el cultivo de alcachofa y papa para obtener mejor rentabilidad. [Tesis]. Huancayo: Facultad de Contabilidad, Universidad Nacional del Centro del Perú; 1992.

4. Sánchez J. Cítricos o Agrios. Chanchamayo: Ministerio de la Presidencia Proyecto Especial Pichis Palcazo; 1999.

5. Promotor del Desarrollo Frutícola Nacional. El cultivo de los cítricos, aspecto de producción, manejo post cosecha y comercialización. Lima; Instituto de Investigación Agraria Comisión Nacional de Fruticultura. Boletín técnico $\mathrm{N}^{\circ} 6$; 1996.

6. La Revista Agraria. El Reto de las Cadenas productivas. 2001; 3 (29):12-13.

7. Urrelo R. Resumen del curso Internacional de Post cosecha de frutas y hortalizas: Universidad Nacional Agraria de la SelvaUniversidad de Costa Rica; 1993.

8. Ponce R. Elementos para el estudio del desarrollo rural: Puno Universidad Nacional del Altiplano; 1999.

E-mail: angelicacastro17@hotmail.com 